\title{
Optimization of Adaptive MTI Filter
}

\author{
Wenxu Zhang, Shudi Ma, Qiuying Du \\ College of Information and Communication Engineering, Harbin Engineering University, Harbin, China \\ Email:mashudi@hrbeu.edu.cn
}

How to cite this paper: Zhang, W.X., Ma, S.D. and Du, Q.Y. (2017) Optimization of Adaptive MTI Filter. Int. J. Communications, Network and System Sciences, 10, 206-217.

https://doi.org/10.4236/ijcns.2017.108B022

Received: May 31, 2017

Accepted: August 11, 2017

Published: August 14, 2017

\begin{abstract}
Moving target indication (MTI) is an effective means for radar to find moving targets in clutter environment. This paper introduces the basic principles of MTI, how to avoid the blind speed problem and the optimization of MTI filter. Implementing the multi-notch adaptive moving target indication (AMTI) filter that designed by using the stagger code in varied cases, which is based on a feature vector method optimization.
\end{abstract}

\section{Keywords}

Adaptive Moving Target Indication (AMTI), Stagger Code, Feature Vector Method, Multi-Notch

\section{Introduction}

MTI band-stop filter as a "single channel", followed by detection is relatively simple. When the target speed is large and the repetition frequency is low, make sure that there is no distance blur, through the "variable week" variable repeat cycle or repeat and "time varying" [1]. Can overcome the blind speed problem, the drawback is no improvement in noise. In general, the mess is not very strong, the radar can handle a limited number of pulses, suitable for the use of repetitive and time-varying weighted system. The adaptive has a variety of ways to achieve, in which the performance is better "first order" and "second order". The firstorder basic method is to use the interval-based velocity measurement and the zero-point distribution method to determine the weighting parameters of the clutter cancellation filter to obtain the filter whose notch is aligned with the center of the clutter spectrum [2]. Its advantages are simpler, the disadvantage is that it cannot be adaptive with the clutter spectrum, so sometimes the performance is worse. The second-order basic method is to estimate the clutter covariance matrix, and then use matrix inversion or feature decomposition feature vector method to determine the filter weight coefficient.

This paper first analyzes the moving target indication (MTI), on this basis, the 
MTI is optimized, and the appropriate filter coefficients are designed by the feature vector method, which can effectively suppress the clutter. And the use of stagger code design MTI filter to eliminate the impact of blind speed. For motion clutter, the spectral center is not at zero frequency, and is time-varying. In order to suppress such clutter, this paper adopts adaptive motion clutter suppression technique AMTI, and designs multi-notch AMTI filter [3].

\section{Research on Adaptive Clutter Suppression Algorithm}

The earliest MTI filter is a delay line canceller, is currently one of the most commonly used MTI filter. According to the different number of cancellation, but also divided into single delay line canceller, double delay line canceller and multi-delay line canceller [4].

Single delay line canceller as shown in Figure 1, the impulse response of the single delay line canceller is expressed as $h(t)$, and output $y(t)$ is equal to the convolution between the impulse response $h(t)$ and the input $x(t)$ [5].

The impulse response of the counter is:

$$
h(t)=\delta(t)-\delta\left(t-T_{r}\right)
$$

The power gain of the single delay line canceller is:

$$
|H(\omega)|^{2}=4\left(\sin \left(\frac{\omega T_{r}}{2}\right)\right)^{2}
$$

Double delay line canceller as shown in Figure 2. The response of the double delay line canceller is

$$
h(t)=\delta(t)-2 \delta\left(t-T_{r}\right)+\delta\left(t-2 T_{r}\right)
$$

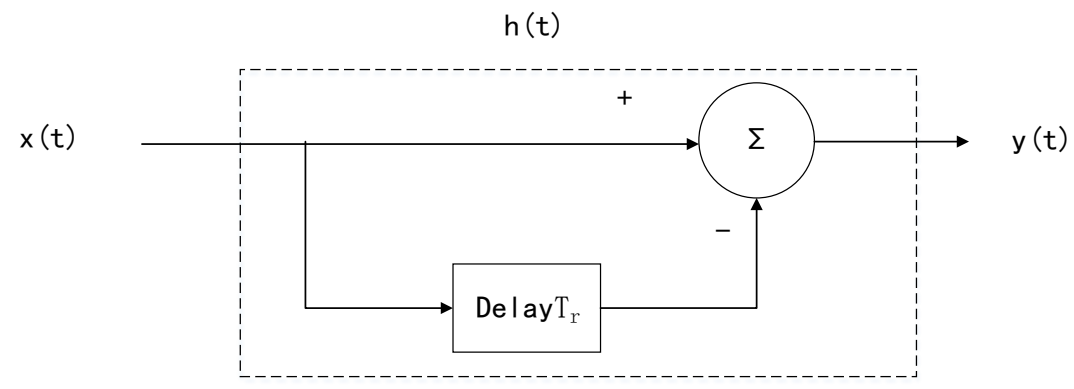

Figure 1. Single delay line canceller.

$\mathrm{h}(\mathrm{t})$

$x(t)$

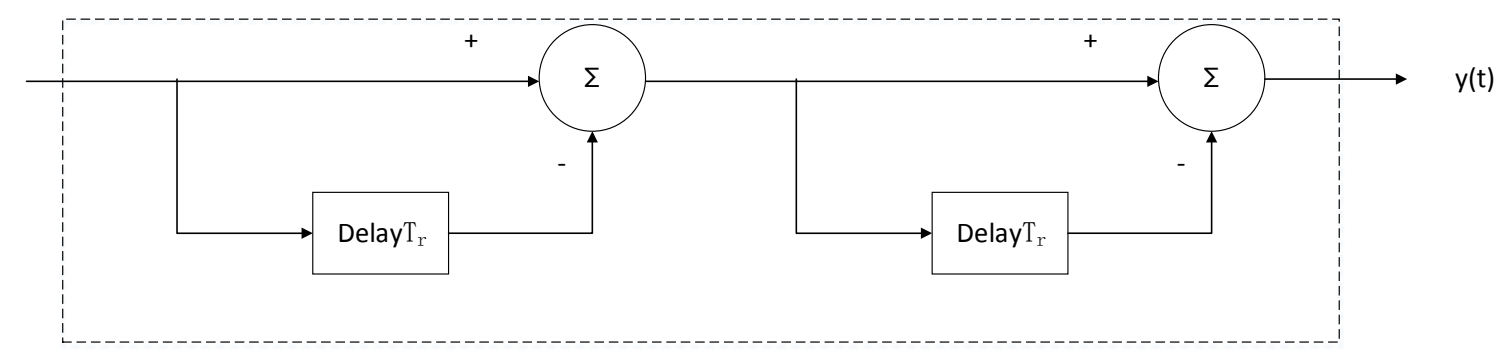

Figure 2. Double delay line canceller. 
The double delay line canceller impulse response is:

$$
|H(\omega)|^{2}=\left|H_{1}(\omega)\right|^{2}\left|H_{1}(\omega)\right|^{2}=16\left(\sin \left(\frac{\omega T_{r}}{2}\right)\right)^{4}
$$

The adaptive moving target indication (AMTI) filter is usually composed of a FIR filter with a horizontal structure. The output of the MTI filter is:

$$
Y(n)=W^{T} X(n)=\sum_{i=0}^{N-1} w_{i} x(n-i)
$$

where $W$ is the weight vector and $X(n)$ is the input signal vector. The frequency response of this filter is:

$$
H(f)=\sum_{i=0}^{N-1} w_{i} \exp \left(-j 2 \pi f T_{i}\right)
$$

In the radar system, in order to avoid the occurrence of blind effects, usually the use of "variable T" approach, that is, by regularly changing the radar launch pulse period so that the frequency of blindness is greater than the target possible Doppler frequency. Adaptive clutter suppression is compatible with parametric techniques, meaning that the clutter suppression filter must be time-varying. For the determined $N$ value, the frequency characteristic of the MTI filter is determined only by the weight vector, so the calculation of the weight vector is the core of the MTI process, according to different design methods, the optimal weight vector is generally different. In engineering practice, the improvement factor is often used to measure the performance of MTI system. The improvement factor of the MTI filter is defined as $I=\left(S_{o} / C_{0}\right) /\left(S_{i} / C_{i}\right)$. Obviously, the greater the $I$, the better the effect of the system on clutter suppression. It has been proved that the optimal weight vector of the MTI filter should be the eigenvector corresponding to the minimum eigenvalue of the covariance matrix of the input clutter, in order to maximize the average improvement factor of the MTI. At this point the improvement factor is $I_{\max }=1 / \lambda_{\min } \quad$ [6].

\subsection{Optimal Design of Filter}

The so-called optimization design requires a set of optimal filter coefficients, to maximize the improvement factor, a lot of design methods. In the case of the variable $\mathrm{T}$, the better methods are feature vector method, matching algorithm, zero-point allocation method and linear prediction method [7]. The feature vector method is the solution that minimizes the clutter output power when the target gain is constant. The zero-point assignment method is to set the frequency response zero at the notch when designing the band-stop filter. The matching algorithm and the linear prediction method are the solutions that minimize the clutter output power when one of the elements of the weight vector is constant. So the feature vector method has better performance [8].

The feature vector method is a clutter suppression method based on the maximum improvement factor.

It is usually assumed that the clutter has a Gaussian power spectrum, the spectral center is $f_{0}$, the spectral width is $\sigma_{f}$, and the spectral density function 
is:

$$
C(f)=\frac{1}{2 \pi \sigma_{f}} \exp \left(-\frac{\left(f-f_{0}\right)^{2}}{2 \sigma_{f}^{2}}\right)
$$

According to the Wiener filter theory, if the clutter is a stationary stochastic process, its power spectrum and autocorrelation function are Fourier transform pairs. Therefore, the clutter autocorrelation function $r_{c}(m, n)$ is the Fourier transform of its power spectrum $C(f)$.

$$
\begin{aligned}
r_{c}(m, n) & =\int_{-\infty}^{+\infty} C(f) e^{j 2 \pi f\left(t_{m}-t_{n}\right)} d f \\
& =\int_{-\infty}^{+\infty} \frac{1}{2 \pi \sigma_{f}} \exp \left[-\frac{\left(f-f_{0}\right)^{2}}{2 \sigma_{f}^{2}}\right] e^{j 2 \pi f\left(t_{m}-t_{n}\right)} d f
\end{aligned}
$$

$\tau_{m n}=t_{m}-t_{n}$ is the relevant time. If the center of the clutter spectrum is zero, then

$$
r_{c}(m, n)=e^{-2 \pi^{2} \sigma^{2} \tau^{2}{ }^{2} m}
$$

We obtain the clutter autocorrelation matrix $A$ of $N$ pulses

$$
R_{c}=\left[\begin{array}{cccc}
r_{c}(0,0) & r_{c}(0,1) & \cdots & r_{c}(0, N-1) \\
r_{c}(1,0) & r_{c}(1,1) & \cdots & r_{c}(1, N-1) \\
\vdots & \vdots & \ddots & \vdots \\
r_{c}(N-1,0) & r_{c}(N-1,1) & \cdots & r_{c}(N-1, N-1)
\end{array}\right]
$$

$B \gg f_{r}$, the Doppler spectrum $S(f)$ of the target echo signal can be expressed as

$$
S(f)=\left\{\begin{array}{c}
1,-\frac{B}{2} \ll f \ll \frac{B}{2} \\
0, \quad \text { other }
\end{array}\right.
$$

The target autocorrelation function is

$$
\begin{aligned}
r_{s}(m, n) & =\frac{1}{B} \int_{-B / 2}^{B / 2} e^{j 2 \pi f \tau_{m n}} d f=\frac{1}{j 2 \pi B \tau_{m n}}\left[e^{j 2 \pi B \tau_{m n} / 2}-e^{-j 2 \pi B \tau_{m n} / 2}\right] \\
& =\frac{\sin \left(\pi B \tau_{m n}\right)}{\pi B \tau_{m n}}= \begin{cases}1, & m=n \\
0, & m \neq n\end{cases}
\end{aligned}
$$

Assume that the clutter data and the target data of the $N$ pulse MTI input are respectively

$$
\begin{gathered}
C=\left[c\left(t_{1}\right), c\left(t_{2}\right), \cdots, c\left(t_{N}\right)\right]^{T} \\
S=\left[s\left(t_{1}\right), s\left(t_{2}\right), \cdots, s\left(t_{N}\right)\right]^{T}
\end{gathered}
$$

Then the MTI output of the clutter power and signal power are

$$
\begin{gathered}
C_{0}=E\left[\left|w^{H} C\right|^{2}\right]=C_{i} w^{H} R_{c} w \\
S_{0}=E\left[\left|w^{H} S\right|^{2}\right]=S_{i} w^{H} R_{s} w
\end{gathered}
$$

where $C_{i}$ and $S_{i}$ represent the clutter power and the signal power at the MTI 
filter input, respectively, $w$ is the weight vector of the FIR filter. According to the definition of the improvement factor of the MTI filter

$$
I=\frac{S_{o} / S_{i}}{C_{o} / C_{i}}=\frac{S_{o}}{S_{i}} \times \frac{C_{i}}{C_{o}}=\frac{S_{i} w^{H} R_{s} w}{S_{i}} \times \frac{C_{i}}{C_{i} w^{H} R_{c} w}=\frac{w^{H} R_{s} w}{w^{H} R_{c} w}
$$

By $r_{s}(m, n)$ know, $R_{s}$ for the unit array, therefore,

$$
I=\frac{w^{H} w}{w^{H} R_{c} w}
$$

The characteristic equation of $R_{c}$ is

$$
R_{c} w_{n}=\lambda_{n} w_{n}, \quad n=0,1, \cdots, N
$$

where $w_{n}$ is the eigenvector corresponding to the eigenvalue $\lambda_{n}$. Among them

$$
\lambda_{0} \ll \lambda_{1} \ll \cdots \ll \lambda_{n}
$$

In the eigenvalues of $R_{c}$, the subspace of the eigenvector corresponding to the large eigenvalue is the subspace of the signal, and the main points of the clutter are located in this subspace. The subspace of the eigenvector corresponding to the small eigenvalue is the noise subspace. Since the noise subspace is orthogonal to the signal subspace, the eigenvector B corresponding to the minimum eigenvalue $\lambda_{0}$ is taken as the weight vector $w_{0}$ of the MTI filter, this can suppress the clutter component to the greatest extent, which is the biggest improvement factor [9].

\subsection{Stagger Repetition Frequency}

In general, it is not possible to obtain a PRF that can meet the required ambiguous distance and Doppler coverage. Therefore, a method of stagger repetition frequency is proposed. Stagger repetition frequency is a measure that can be used to prevent blind influence [10].

If the radar uses $N$ repetition frequencies, their repetition periods can be expressed as

$$
\left\{\begin{array}{c}
T_{r 1}=1 / f_{r 1}=K_{1} \Delta T \\
T_{r 2}=1 / f_{r 2}=K_{2} \Delta T \\
\cdots \\
T_{r N}=1 / f_{r N}=K_{N} \Delta T
\end{array}\right.
$$

$\Delta T$ is the maximum convention period for $\left[T_{r 1}, T_{r 2}, \cdots, T_{r N}\right]$, then the odds ratio is:

$$
T_{r 1}: T_{r 2}: \cdots: T_{r N}=K_{1}: K_{2}: \cdots: K_{N}
$$

$\left[K_{1}: K_{2}: \cdots: K_{N}\right]$ is the stagger code, the ratio of the largest $K$ value to the minimum $K$ value in the parametric code is called the maximum ratio $r$ of the azimuth cycle.

$$
r=\max \left[K_{1}: K_{2}: \cdots: K_{N}\right] /\left[K_{1}: K_{2}: \cdots: K_{N}\right]
$$

If $K_{i}$ is mutually different and satisfies Equation (22), then the first true blind velocity corresponds to the Doppler frequency $f_{b n}$. 


$$
f_{b n}=\frac{1}{\Delta T}
$$

The average repetition period of the radar is

$$
T_{r}=\frac{1}{N} \sum_{i=1}^{N} T_{r i}=K_{a v} \Delta T
$$

$K_{a v}$ is the mean of the difference. Therefore

$$
\begin{gathered}
K_{a v}=\frac{T_{r}}{\Delta T}=T_{r} f_{b n}=\frac{f_{b n}}{f_{r}} \\
f_{b n}=K_{a v} f_{r}
\end{gathered}
$$

Because $f_{r}=1 / T_{r}$ is the average radar repetition frequency, it is also called $K_{a v}$ for the blind expansion factor.

The coefficient of the MTI filter between the pulses is different for each pulse of the three pulse canceller, so it is a time-varying filter. If the radar uses three repetition frequencies $T_{1}, T_{2}, T_{3}$ at one time, three sets of MTI filters work in turn. The depth of the stagger MTI filter speed response notch is independent of the form of the canceller and is independent of the pulse received in the radar antenna beam and is related to the maximum ratio of the azimuth cycle. The larger the maximum change ratio, the shallower the corresponding notch depth.

\subsection{Optimization of Adaptive MTI Filter}

In the clutter region, the spectral center $f_{d}$ of the motion clutter in the input signal is estimated to obtain the Doppler frequency $f_{d}$ estimate of the center of the clutter spectrum. And then estimate the spectral width $B$ to obtain the estimated value $\hat{B}$ of the spectral width. Then we obtain the weight coefficient of the multi-notch filter by using the obtained estimator $\hat{f}_{d}$ and $\hat{B}$ into the feature vector method, and design the MTI filter with multi-notch. As shown in

\section{Figure 3.}

First estimate the motion of the clutter spectrum center.

The radar suffers from narrowband clutter and noise that can be expressed as

$$
u(t)=A(t) e^{j\left(\omega_{d} t+\varphi_{0}\right)}+n(t)
$$

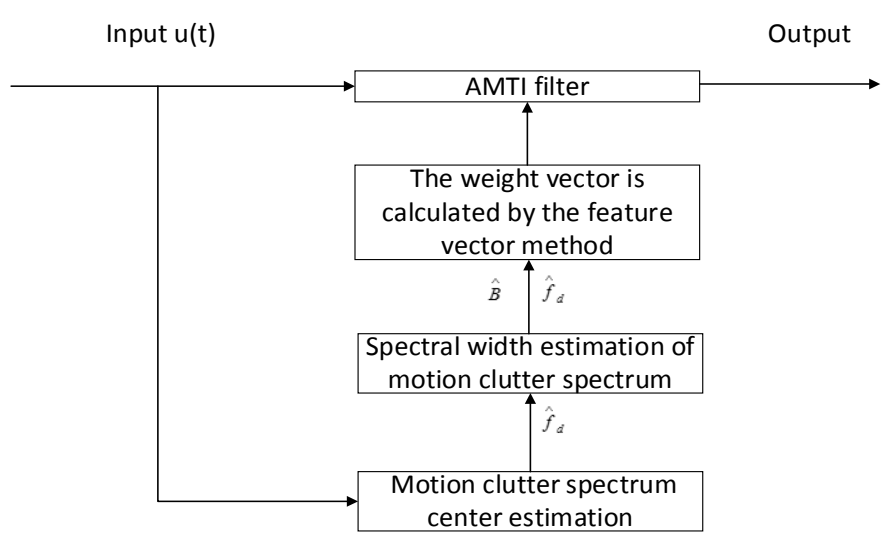

Figure 3. Optimization design of adaptive MTI filter. 
$A(t)$ is the amplitude, $\omega_{d}$ is the Doppler frequency of the clutter, $\varphi_{0}$ is the initial phase, and $n(t)$ is the additive noise. Noise is not related to clutter, and noise between different PRI is uncorrelated.

Delay the signal after a PRI

$$
u\left(t-T_{r}\right)=A\left(t-T_{r}\right) e^{j\left(\omega_{d}\left(t-T_{r}\right)+\varphi_{0}\right)}+n\left(t-T_{r}\right)
$$

The correlation function of $u(t)$ and $u\left(t-T_{r}\right)$ is

$$
R\left(T_{r}\right)=E\left[u(t) u^{*}\left(t-T_{r}\right)\right]=E\left[A(t) A\left(t-T_{r}\right)\right] e^{j \omega_{d} T_{r}}
$$

Therefore, the center frequency estimate of the clutter spectrum is obtained

$$
\hat{f}_{d}=\frac{1}{2 \pi T_{r}} \arctan \frac{\operatorname{Im}\left[\hat{R}\left(T_{r}\right)\right]}{\operatorname{Re}\left[\hat{R}\left(T_{r}\right)\right]}
$$

After obtaining the center frequency of the clutter spectrum, the spectral width estimation is performed by the integral method.

Combined with the Gauss spectrum, there are Gaussian power spectra

$$
C(f)=P_{c} \frac{1}{\sqrt{2 \pi} \sigma_{f}} \exp \left[-\frac{\left(f-f_{d}\right)^{2}}{2 \sigma_{f}^{2}}\right]
$$

$\sigma_{f}$ is the frequency variance of the Gaussian power spectrum, $f_{d}$ is the center of the power spectrum, and $P_{c}$ is the corresponding power spectrum at zero Doppler frequency. According to the definition of half power points $\Delta f_{\text {зdB }} \cong 2.355 \sigma_{f}$.

According to the nature of Gaussian distribution, there are

$$
\left\{\begin{array}{c}
P\{\mu-\sigma<x \leq \mu+\sigma\}=\Phi(1)-\Phi(-1)=0.6826 \\
P\{\mu-2 \sigma<x \leq \mu+2 \sigma\}=\Phi(2)-\Phi(-2)=0.9544 \\
P\{\mu-3 \sigma<x \leq \mu+3 \sigma\}=\Phi(3)-\Phi(-3)=0.9974
\end{array}\right.
$$

Prior to the estimated spectrum as the center to both sides of the center $\hat{f}_{d}$ of the accumulated clutter power spectrum (corresponding to integration), to 95.44\% for the energy threshold, and then using the relationship between $\Delta f_{3 \mathrm{~dB}}$ and $\sigma_{f}$ to the spectral width $\hat{B}$ of the spectral estimate Gauss. After obtaining the estimated spectral center and estimating the spectrum width, the weight coefficient of the filter is obtained by using the feature vector method.

It is found that the power spectrum is the sum of their respective power spectra for the stagger clutter of multiple Gaussian spectra. The autocorrelation function should also have the sum of the corresponding multi-clutter components. Thus, we can derive the weight coefficients of two or more notch filters to design a multi-notch AMTI filter.

\section{Simulation and Performance Analysis}

In Figure 4, obviously, the frequency response of the single delay line canceller and the double delay line canceller changes cyclically, and the period is $f_{r}$. The peak appears at $f=(2 n+1) /\left(2 f_{r}\right)$, and the zero value appears at $f=n f_{r}, n \geq 0$. As 


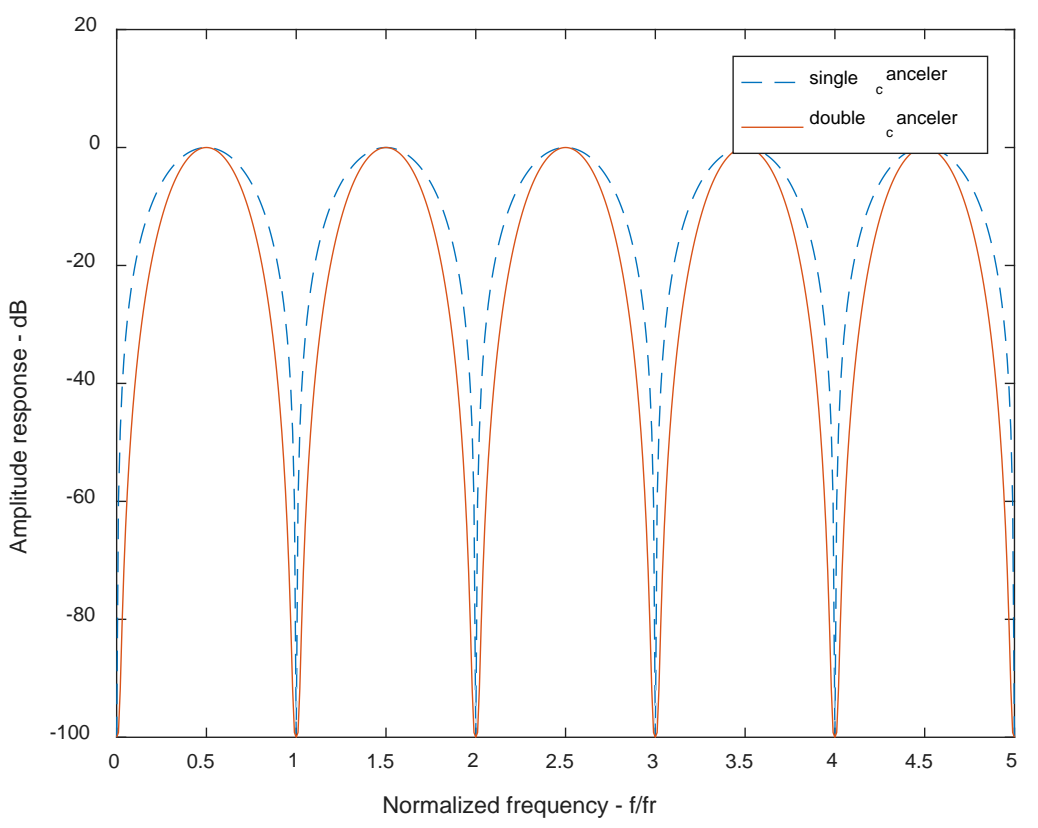

(a)

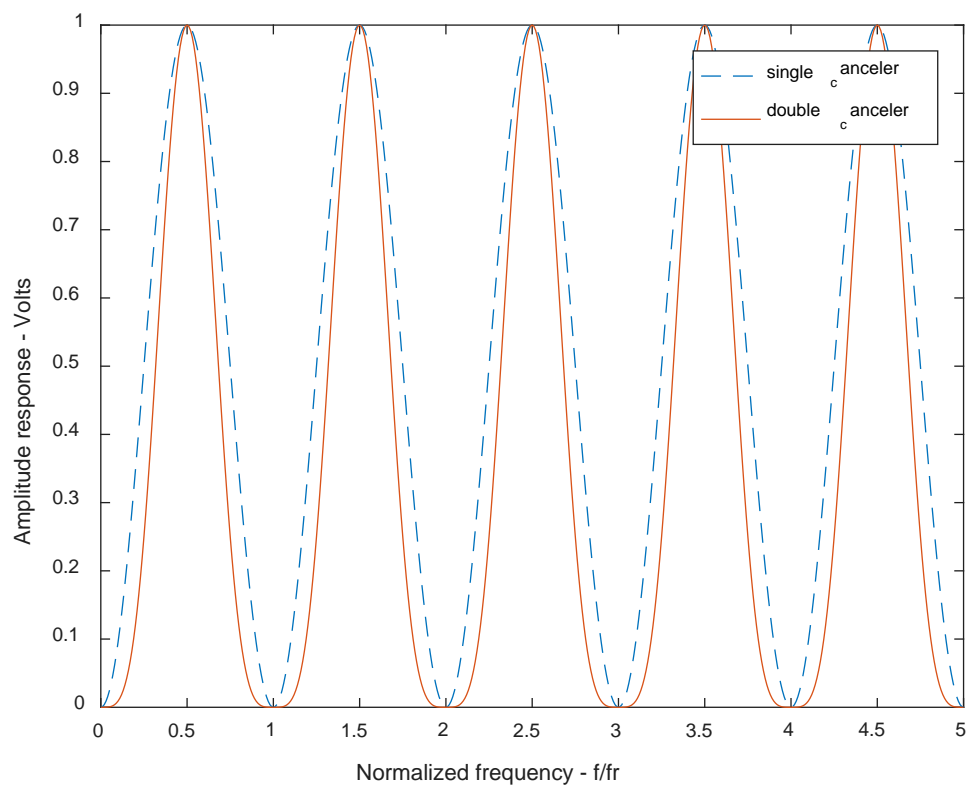

(b)

Figure 4. Normalized frequency response of single delay line canceller and double delay line supporter. (a) dB. (b) Volt.

can be seen from the figure, the double delay line canceller has a deeper notch and a more flat passband response than a single delay line canceller.

In Figure 5, the frequency response is still cyclical when the $\mathrm{T}$ is equal. It can be clearly seen from the figure that the notch depth is significantly enhanced compared to the delay line canceller, the passband response is also more flat, and the frequency of the notches can be set at the same time.

In Figure 6, it can be seen that the use of staggered repetition frequency can greatly improve the first blind speed. The larger stagger ratio, the lighter the 


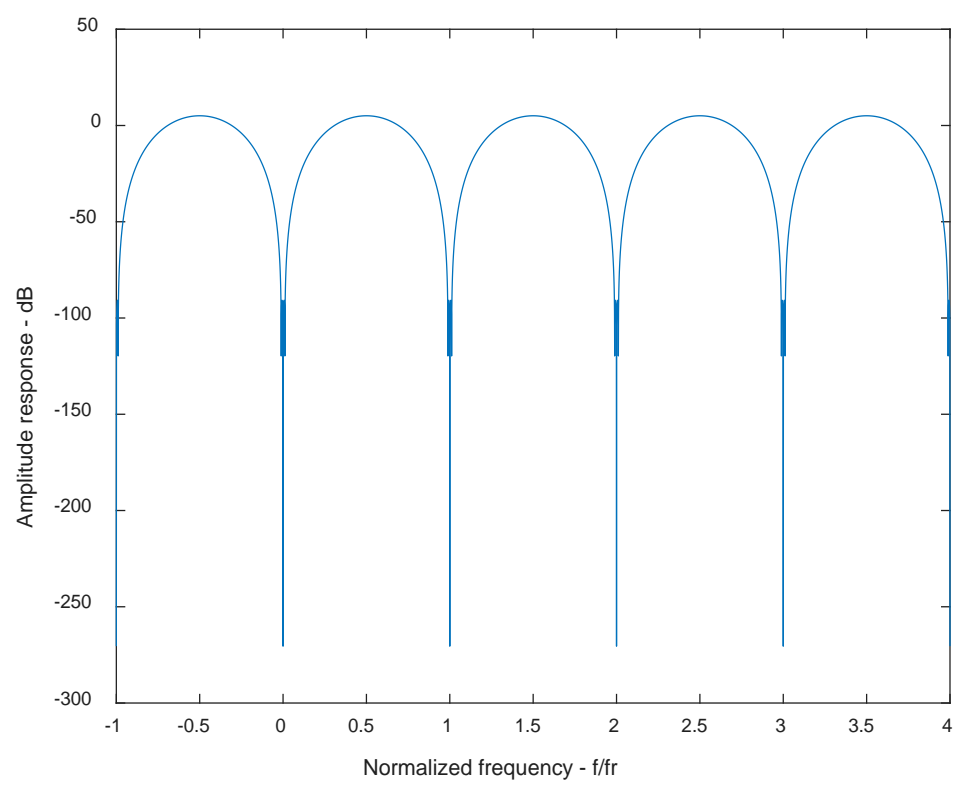

(a)

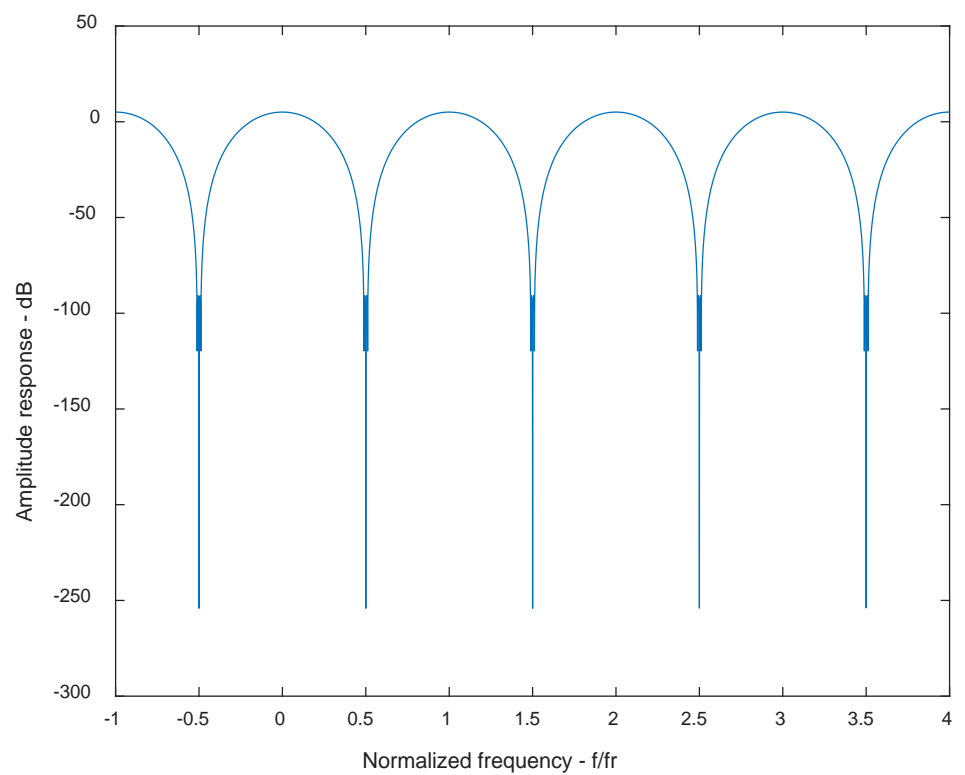

(b)

Figure 5. Normalized frequency response of the MTI filter. (a) Center of the clutter spectrum: $0 \mathrm{~Hz}$. (b) Center of the clutter spectrum: $50 \mathrm{~Hz}$.

corresponding notch, and avoid the loss of weak targets in one of them.

Figure 7 shows the normalized frequency response of the MTI filter designed using the feature vector method, Filter length of 4 order, the average pulse repetition frequency of $100 \mathrm{~Hz}$, the stagger ratio of 15:16:17, The center of the clutter spectrum is selected as $0 \mathrm{~Hz}$ and $50 \mathrm{~Hz}$, respectively, the spectral width is $0.64 \mathrm{~Hz}$. The filter has a very deep notch at the clutter component, the entire pass band is relatively flat, and effectively suppresses the blind speed.

In Figure 8, filter length of 4 order, the average pulse repetition frequency of $100 \mathrm{~Hz}$, the stagger ratio of 15:16:17. The clutter center frequency is $0 \mathrm{~Hz}$, the 


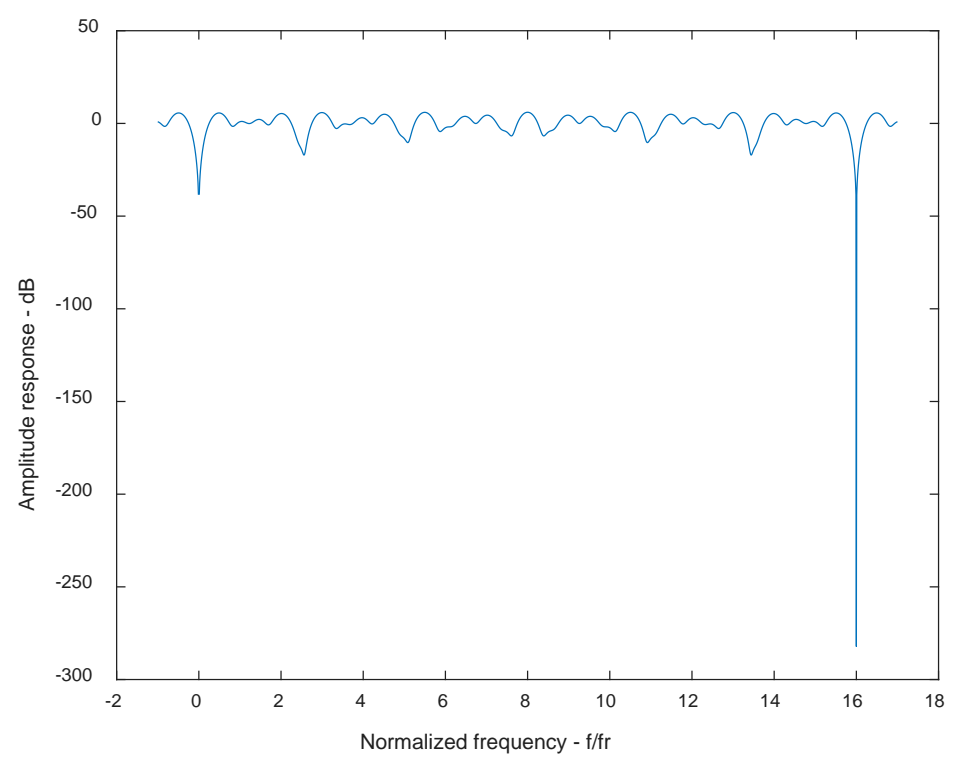

(a)

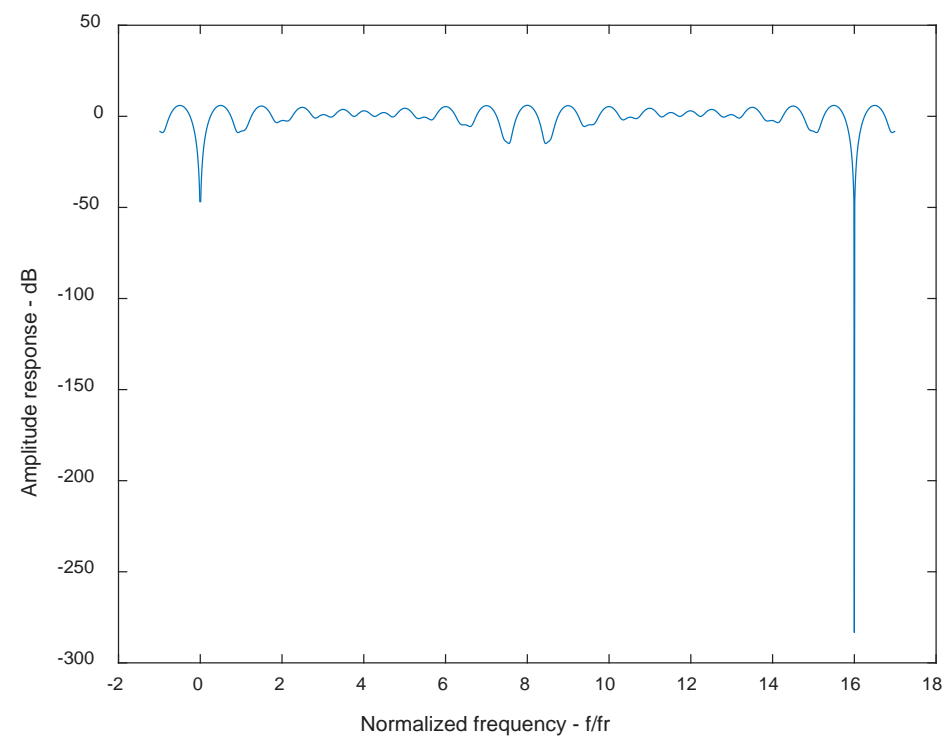

(b)

Figure 6. Normalized frequency response of the three-pulse differential register. (a) Stagger ratio: 13:16:19. (b) Stagger ratio: 15:16:17.

spectral width is $0.64 \mathrm{~Hz}$, the meteorological clutter center frequency is $30 \mathrm{~Hz}$, the spectral width is $1.4 \mathrm{~Hz}$. It can be seen from the figure at $0 \mathrm{~Hz}$ and $30 \mathrm{~Hz}$ with a deeper notch, can inhibit the clutter.

\section{Conclusion}

In the process of receiving the echo signal by the radar, the presence of the clutter signal has been interfering with the detection and extraction of the useful signal, it is necessary to suppress clutter. The moving target indication (MTI) technique has a good ability to suppress static clutter, but it is powerless for dynamic clutter. The use of adaptive technology can effectively inhibit the dynamic 


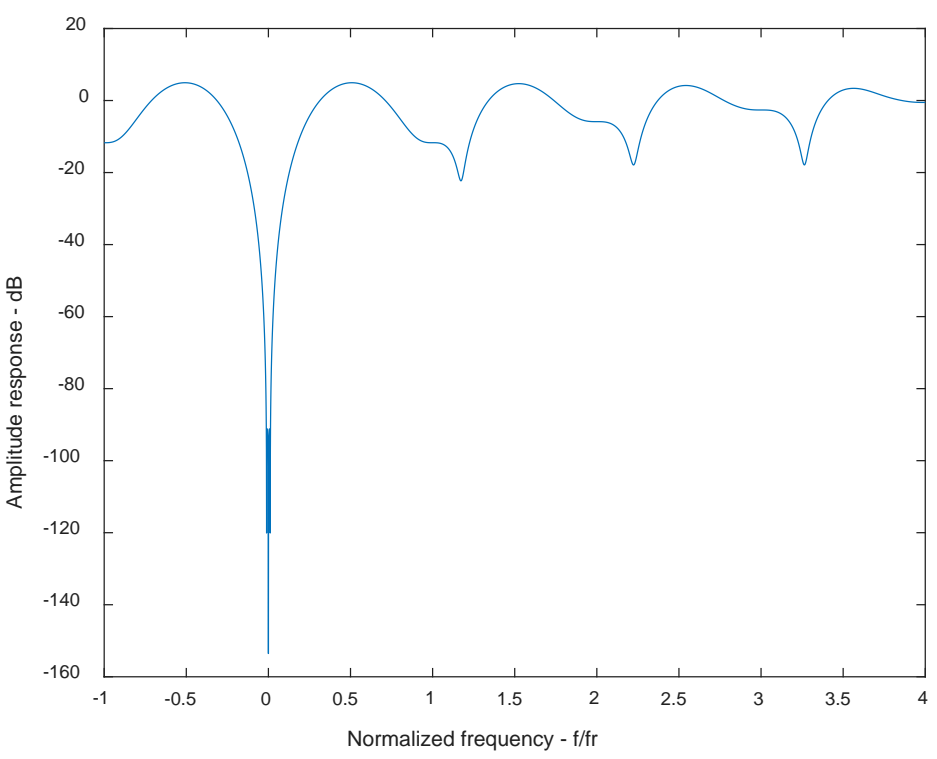

(a)

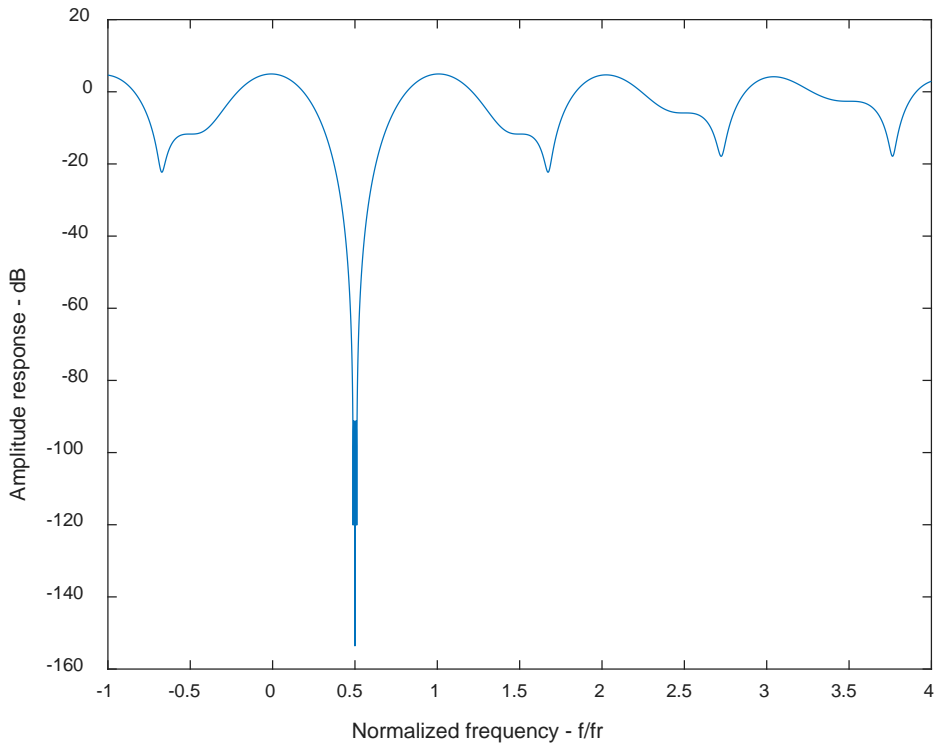

(b)

Figure 7. Normalized frequency response for the 15: 16: 17MTI filter. (a) Center of the clutter spectrum: $0 \mathrm{~Hz}$. (b) Center of the clutter spectrum: 50 $\mathrm{Hz}$.

clutter. In this paper, we propose an algorithm for processing AMTI based on the maximum average improvement factor, and give the corresponding MATLAB simulation waveform. Especially with the development of DSP chip, the processing speed has been improved, which made this method very suitable for practical application.

\section{Acknowledgements}

This work is supported partly by National Natural Science Foundation of China under Grant No. 61301205 and No. 61571146, National Defense Based Science 


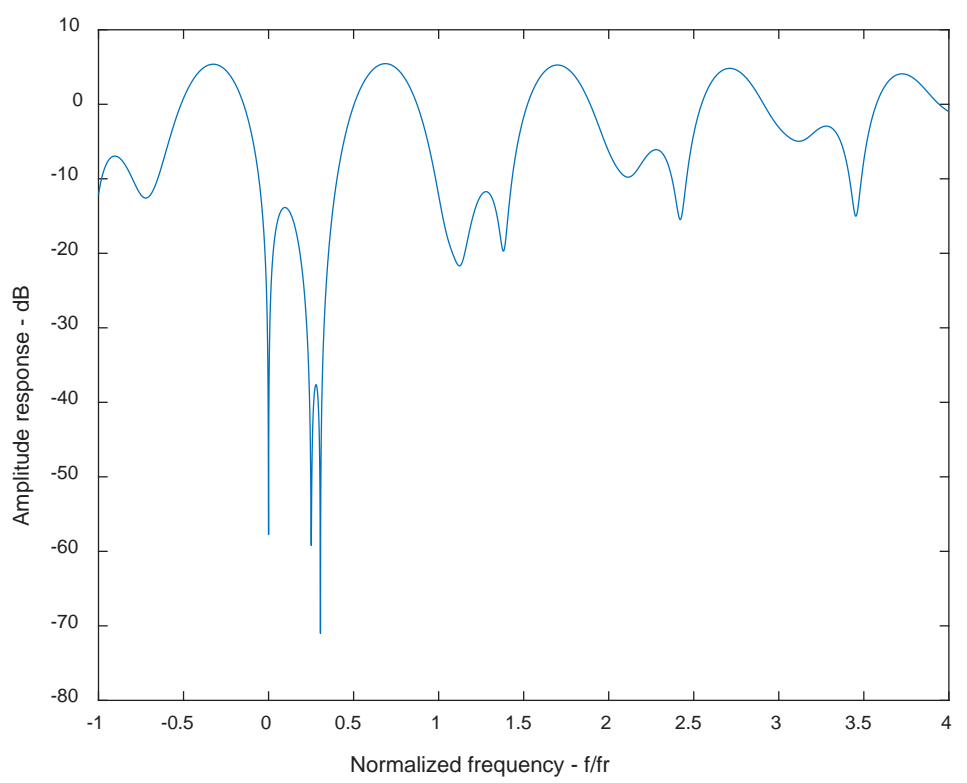

Figure 8. Normalized frequency response of multi-notch adaptive MTI (AMTI).

Research Program under Grant No. JCKY2013604B001. This paper is funded by the International Exchange Program of Harbin Engineering University for Innovation-oriented Talents Cultivation.

\section{References}

[1] Geng, F.L. (2006) Radar Principle. Xi'an University of Electronic Science and Technology Press, Xi'an.

[2] Wu, S.J. and Mei, X.C. (2008) Radar Signal Processing and Data Processing Technology. Electronic Industry Press, Beijing, 106-134.

[3] Yuan, B.H., Zhang, W.X. and Zhong, X.K. (2017) Reconfigurable Multi-Channel Radar Transmitter Based on SDR. Applied Science and Technology.

[4] Chen, J.C. and Geng, F.L. (1999) An Adaptive Moving Clutter Rejection Technique. $J X i$ an Electronics Technology University, 26, 174-177.

[5] Yang, R. (2012) For the Cognitive Radar Waveform and Staggered Filter Optimization Algorithm. Xi'an University of Electronic Science and Technology.

[6] Sun, C., Li, M. and Tao, H.H. (2014) Design of Gradient MTI Filter Based on Gradient Immune Algorithm. Journal of China Institute of Electronic Science and Technology, 1673-5692.

[7] Malanowski, M. (2006) Comparison of Adaptive Methods for Clutter Removal in PCL Radar. International Radar Symposium, Krakow, 24-26 May 2006, 1-4. https://doi.org/10.1109/IRS.2006.4338044

[8] Hu, L.X. (2013) Application of EMD Algorithm in Radar Clutter Suppression. Xi'an University of Electronic Science and Technology.

[9] Zhu, W. (2010) Research and Implementation of MTI \& MTD in Some VHF Radar. Xi'an University of Electronic Science and Technology.

[10] Hu, K.X. (2006) Application of an Adaptive Clutter Rejection Technique in Radar. Modern Electronics Technique, 29, 24-26. 
Submit or recommend next manuscript to SCIRP and we will provide best service for you:

Accepting pre-submission inquiries through Email, Facebook, LinkedIn, Twitter, etc. A wide selection of journals (inclusive of 9 subjects, more than 200 journals)

Providing 24-hour high-quality service

User-friendly online submission system

Fair and swift peer-review system

Efficient typesetting and proofreading procedure

Display of the result of downloads and visits, as well as the number of cited articles Maximum dissemination of your research work

Submit your manuscript at: http://papersubmission.scirp.org/

Or contact ijcns@scirp.org 